\title{
INVESTIGACIONES
}

\section{Introyección de saberes metodológicos transpuestos en los trabajos de titulación de grado*}

\author{
Methodological knowledge introjection transposed in the final thesis for graduation \\ Introjecção dos saberes metodológicos transpostos \\ nos trabalhos de colação de grau \\ Tomás Fontaines R., ${ }^{a}$ Italo Jiménez I. ${ }^{b}$ \\ aDoctor en Ciencias Humanas. Investigador Prometeo-SENESCYT, Universidad Técnica de Machala. \\ Universidad de Oriente, Venezuela \\ Correo electrónico: tfontaines@hotmail.com

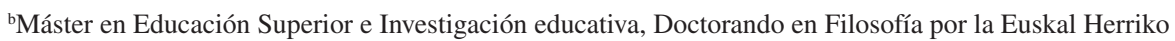 \\ Unibertsitatea (UPV/EHU). Profesor de la Universidad Técnica de Machala. \\ Correo electrónico: italosj@ hotmail.com
}

\begin{abstract}
RESUMEN
Con el objetivo de describir los introyectos identificados a partir del análisis de contenido aplicado a las secuencias operativas de investigación subyacentes en los trabajos de titulación presentados en la Unidad Académica de Ciencias Sociales de la Universidad Técnica de Machala, Ecuador, se emprendió la presente investigación descriptivo-documental, cuya unidad de análisis fueron 220 trabajos de titulación presentados en la precitada unidad académica durante el año 2014, corpus al que se realizó análisis de contenido. Se concluyó que los trabajos de titulación son el resultado físico de los siguiente introyectos metodológicos: a) El método está por encima de las diferencias estructurales y funcionales del objeto, b) Imposición arquetípica de la dirección de las variables, c) Los datos se deben evaluar estadísticamente para garantizar cientificidad en el estudio, d) Valoración sesgada del paradigma, toda investigación debe llevar aparejada una propuesta.
\end{abstract}

Palabras clave: introyectos metodológicos, transposición didáctica, formación de noveles investigadores, trabajos de titulación.

\begin{abstract}
The following descriptive and documental research was undertaken in order to describe the introjections identified from content analysis applied to the operational sequences underlying research works presented to get the degree at the Social Sciences Academic Unit of the Technical University of Machala, Ecuador. The unit of analysis consisted of 220 degree works presented in the aforementioned academic unit during 2014. We made content analysis of the corpus. It was concluded that the research degree works are the physical result of the following methodological introjections: a) The method is above the structural and functional differences of the object, b) Archetypal imposition of the orientation of the variables, c) Data must be statistically evaluated to guarantee scientific quality in the study, d) Biased paradigm evaluation, all research must be accompanied by a proposal.
\end{abstract}

Key words: methodological introjections, didactic transposition, novice researcher training, university degrees.

Agradecemos al Proyecto Prometeo de la Secretaría Nacional de Educación Superior, Ciencia, Tecnología e innovación de la República del Ecuador por su patrocinio en este trabajo. 


\section{INTRODUCCIÓN}

Al estudiar la estructura y funcionamiento de la formación de investigadores en la universidad, identificamos fluctuaciones entre perspectivas que van desde la apertura conceptual crítica, respecto al abordaje del diseño e instrumentalización de los procesos metodológicos, hasta la imposición de esquemas procedimentales inviolables como condición para el reconocimiento y aprobación del producto desarrollado, sin distingo del género textual en el que se exprese.

Esta variación sugiere que en los centros universitarios el saber metodológico es objeto de la transposición (Caparrós, 2012; Johsua y Dupin, 2005) que empieza siendo didáctica y tiende a convertirse en dogmática como resultado de la introyección no consciente de los saberes transpuestos. Con base en lo comentado, el presente trabajo se plantea como objetivo describir tales introyectos en las secuencias operativas de investigación subyacentes en los trabajos de titulación presentados en la Unidad Académica de Ciencias Sociales (UACS), de la Universidad Técnica de Machala (UTMach), Ecuador.

Partiendo de lo dicho, el problema subyacente tiene al menos dos aristas o categorías estructurales. La primera de ellas la clasificamos como onto-epistémica y nos muestra que la realidad es objeto de la construcción humana y que la voluntad de poder de quienes deciden condiciona la amplitud o estrechez en la valoración de propuestas metodológicas. La segunda categoría es de naturaleza didáctica y dirige su atención a la comprensión del modo en que opera el binomio transposición-introyección como garante de progresión u obstáculo en el desarrollo de investigaciones. A continuación se desarrolla cada una de ellas.

Iniciaremos identificando en la historia de la ciencia testimonios de las confrontaciones entre pensadores que defienden la cientificidad de sus prácticas, con base en el cumplimiento de determinadas disposiciones epistémicas y metodológicas. Están los que reconocen el empirismo-inductivo representado en los aportes de Bacon, Newton, Comte, Mach y los del Círculo de Viena (Álvarez, 1999; Bunge, 2004; Camacho, 2001; Echeverría, 1995; Padrón, 1998) como el soporte de un saber válido y confiable. También se ubican los que defienden el camino racional-deductivo como el más viable para generar teorías capaces de ser generalizadas (Lakatos, 2007, 2011; Popper, 1972, 1982) alardeando de la incapacidad que tiene la inducción para lograr teorías que den respuestas a los condicionantes universales (todos, siempre, nunca).

En otra dirección, Feyerabend (1986, 2008) nos invita a desconectarnos de las prescripciones metódicas para investigar. Promueve las libertades metodológicas en oposición a la linealidad para comprender el objeto de estudio. Asume que la realidad es plural y, por ende, los caminos para abordarla. Este filósofo impulsa la consigna epistémicometodológica "todo vale", aludiendo a las posibilidades que tiene el investigador para diseñar rutinas metódicas que, enmarcadas en la prudencia metodológica, se correspondan con los objetivos del estudio; hecho que sirvió de soporte diacrónico para la concepción de la mixtura de métodos en las investigaciones (Fontaines, 2010; Henwood, 2005; Hesse, 2010).

En esta muestra no podrían faltar los representes de tendencias que rescatan el énfasis subjetivo (Gadamer, 2007; Husserl, 1998), histórico-crítico (Giroux, 1990; Habermas, 2008; Outhwaite, 1994) y complejo (Prigoggine, 1997, 2009) en la construcción del conocimiento, y que además hacen del sujeto el eje central en la producción del saber devolviéndole el lugar que empiristas y racionalistas le habían negado. 
En lo mostrado se reconoce la investigación como cambiante. Ésta se configura y des-configura con base en la voluntad de poder (Nietzsche, 2000) de quien la dirige, se legitima y deslegitima en atención a los criterios asumidos por la comunidad que la impulsa y la avala. Pero también mostramos acuerdo con la idea de que el objeto antecede a la investigación y que el sujeto lo cualifica (Kant, 2007). Hacemos constar la naturaleza humana de las confrontaciones sobre lo que es investigación y cómo se hace, así como el nexo investigación-poder. Justamente, es esta dialéctica subyacente la que finalmente permite el avance $u$ obstaculiza el progreso de quienes investigan un área de conocimiento con la finalidad de describirla, comprenderla o explicarla según sea la intención del estudio.

También podríamos inferir de las ideas precitadas que la realidad, y por tanto los hechos científicos, son producto de la construcción humana. Se pueden cuestionar, deconstruir y re-construir, en función a los preconceptos de quien los descubra o construya. Esto significa que la ciencia como organización es el resultado de la participación de los actores sociales que normalizan con su acuerdo el criterio de demarcación entre lo cierto y lo incierto, lo científico de lo pseudocientífico y, lógicamente, terminan siendo testimonio del nivel de inteligibilidad de determinados saberes y con ello de la aceptación de los mismos.

Como ejemplo de lo señalado tomaremos el tránsito de la teoría geocéntrica a la heliocéntrica (Santos, 2014; Villacañas, 2013). La primera indica que la tierra se encuentra en una posición fija y que el sol, la luna y las estrellas giran alrededor de ella. Esta verdad fue acuñada en la antigüedad por Aristóteles, Ptolomeo, entre otros griegos, y fortalecida en los escritos de la época medieval. Suponer cambios en este modo de entender el mundo, contravenía la autoridad y ser quemado en la hoguera era un destino seguro, tal como sucedió con Giordano Bruno en el año 1600.

No obstante, muy a pesar de la resistencia de quienes apoyaban lo geocéntrico, sus observaciones anómalas fueron cada vez mayores, dando paso a pensamientos que argumentan en favor de un modo diferente de entender el mundo. Surge en el siglo XVI una postura emergente: la revolución copernicana y, como signo de ella, la teoría heliocéntrica. Esta visión sostiene que el sol está inmóvil en un punto central y la tierra gira alrededor de ella. En el lenguaje de Kuhn (2004) se produce una revolución paradigmática que catapulta las Leyes de Kepler, las investigaciones experimentales de Galileo, convirtiéndose éstas en el semillero para el descubrimiento de la mecánica newtoniana y, con ella, el cambio en la mentalidad del mundo moderno.

En este segmento de historia queremos resaltar cómo los esfuerzos de Galileo, Kepler, Copérnico, Giordano Bruno, entre otros, fueron señales de una situación que siempre ha estado allí. La tierra no empezó a girar alrededor del sol a partir de Copérnico. Siempre lo había hecho. El agregado que en este caso puntualizamos es que el hombre termina fomentando realidades, construyendo verdades y como colectivo las asumimos y vivimos de cara u oposición a ello, tal como sucedió en la cultura occidental con la llegada de la ilustración.

La segunda categoría del problema, vinculada a los procesos didácticos de la investigación, nos alerta sobre la manipulación de los contenidos y el orden en que se enseñan y procesan dentro de un estudio. Si asumimos este supuesto como cierto, entonces estaremos de acuerdo en que cada centro de formación universitaria traspone a voluntad, la perspectiva de la investigación que enseña, los recursos que selecciona para ello, 
los esquemas que guían sus secuencias operativas, cuya consecuencia más palpable es la homogenización del proceso investigativo, sin discriminar el objeto en estudio ni la intencionalidad de los mismos.

Otra de las consecuencias de la introyección del saber metodológico como resultado de la trasposición, es que no estudiamos el saber metodológico desde una perspectiva global. Solo hacemos aprehensión de los contenidos que los tutores, en nombre de los centros de formación, seleccionan para ser enseñados, y luego los formandos terminan construyendo esquemas cognitivos que privilegian el modo de investigar que han aprendido sobre otra posibilidad. Es justamente aquí donde el paradigma de formación pudiera caer en lo dogmático y empieza a tener un carácter mesiánico, como resultado de la alteración de sus fortalezas y la omisión de sus debilidades.

A esta lista agregaremos un fenómeno traído de las ciencias biológicas denominado mimetismo (Bornmann, 2011; Moynihan, 1968; Pasteur, 1982), pero que para los efectos de comprender su inherencia lo adjetivaremos con el término científico. De este modo, el mimetismo científico lo identificamos como la imitación de secuencias operativas de investigación (Camacho, 2001; Fontaines, 2012) de las ciencias dominantes para ser considerados miembros de la comunidad científica. Ejemplo de esta condición la tenemos en la visión comteana de la sociología (física social) y en los componentes del ethos mertoniano, entre otros (León, 2008; Merton, 1996).

Si volteamos la mirada a los modos de enseñar la investigación, el mimetismo científico tiene un lugar privilegiado como consecuencia del culto al paradigma. Cuando la devoción es hacia el paradigma cuantitativo, entonces, las investigaciones válidas seguirán los protocolos metodológicos análogos a los que se usan en biología, química, física, etc. Si por el contrario, el paradigma regente es cualitativo, entonces se exaltarán los procederes antropocéntricos donde predomina lo subjetivo como el eje central de la investigación que se enseña, aprende y ejecuta.

Como se puede percibir, más allá de justificar modos de hacer y enseñar investigación, el riesgo del mimetismo es la estrechez de enfoques e inconsistencias entre el objeto y la metodología empleada. Esto se traduce en que el método se hace autónomo en la transferencia de validez al estudio y, por esto, deja de ser un medio para constituirse en un fin, al imponerse al objeto de estudio en quebranto de su singularidad.

La síntesis del problema sugiere que la voluntad de poder de quienes coordinan y enseñan investigación traspone didácticamente saberes metodológicos que terminan siendo introyectados por los estudiantes, provocando como consecuencia la presencia de inconsistencias metódicas en los trabajos de titulación que testimonian la posesión de concepciones erróneas respecto a la estructura y secuencias operativas de la misma.

Suponemos que al develar el contenido de los introyectos, se crean alertas para el diseño de controles previos y concurrentes en el desarrollo de los estudios conducentes a titulación en la UTMach, previendo de esta manera, inconsistencias de forma y fondo que muchas veces saltan a la vista en detrimento del producto que la ostenta.

Para efectos de la estructura del presente informe, el mismo estará compuesto por tres secciones: en primer término se describen los ejes teóricos que sustentan las variables del estudio: proceso de introyección y trasposición didáctica. Luego se expone el momento metodológico de la investigación, para finalmente dar a conocer los introyectos detectados como resultados del estudio, seguido de las reflexiones finales. A continuación se desarrollan los aspectos indicados. 


\section{DE LA INTROYECCIÓN COMO PROCESO NEURÓTICO A LA INTROYECCIÓN DIFERENCIADA DE SABERES METODOLÓGICOS}

El concepto de introyección tiene su origen en el legado psicoanalítico del húngaro Sándor Ferenczi, durante la primera década del siglo XX (Gutiérrez, 2013). Este constructo designa la incorporación e integración en la estructura yoica de elementos del mundo exterior (González et al., 2005; Sainsbury, 1978). Al igual que Freud, Ferenczi indica que la introyección es opuesta al concepto de proyección y la identifica como constitutiva del superyo, debido a la penetración inconsciente de estándares externos planteados por la sociedad o sus figuras significantes que sucede en el interior del sujeto (Sánchez, 2010).

Al introyectar configuramos internamente objetos y los clasificamos como buenos o malos según la capacidad de mediación que estos tengan para la satisfacción de las necesidades (Sainsbury, 1978). En la primera infancia, el introyecto es clave para identificar y comprender el entorno circundante. Por ejemplo, el niño introyecta el seno materno y lo valora en virtud de la obtención o no de la lactancia. En este acto va cualificando y apropiándose de su experiencia mediante la adopción del sentido que el otro le otorga, dando lugar a la formación del núcleo del yo (Arros y Valenzu, 2006). Por tanto, la introyección también es considerada como un proceso psicológico primario en la organización de los referentes de significación psicológica (Pacheco y Pons, 2003).

Como se puede inferir, es clara la presencia del lenguaje como medio para provocar la introyección. Del mismo modo que Vigotsky (2004) lo responsabiliza de la configuración de los procesos psicológicos superiores, los psicoanalistas se unen a esta voz y reconocen al lenguaje como hacedor del objeto que se introyecta. En tal sentido, la introyección la podemos contactar durante la recepción de opiniones, creencias, pautas conductuales transmitidas por otras personas, siendo esta dinámica un eje articulador de la socialización (Hough, 1999), pero al mismo tiempo puede tener un efecto adverso si el contenido asumido no se comprende de modo crítico y se hace uso consciente de ello.

Lo introyectado se considera como un objeto y la interacción entre esos objetos forma representaciones mentales que llegan a personalizarse al punto de ser utilizadas como referentes para juzgar la realidad (Rycroft, 2010). En cuanto a los modos de integración del introyecto a la estructura de personalidad, este hecho sucede de forma análoga al funcionamiento de las invariantes del pensamiento identificadas en la teoría de Piaget: asimilación-acomodación-adaptación y organización (Fontaines y Rodríguez, 2008), aun cuando no existe correspondencia directa entre las teorías.

Tal como hemos mencionado, la presencia de la introyección va a ser clave en la construcción de la socialización, pero existen situaciones donde la dinámica de llevar al interior elementos del contexto pone en riesgo el límite entre el sujeto individual y el resto del mundo. Cuando la entrada del elemento externo ha sido de un nivel tan profundo, intrusivo y abrumador, el sujeto se desconoce a sí mismo y funciona en congruencia con el objeto introyectado, aunque esto signifique una disociación de su personalidad (Perls, 1997).

En este punto surge una especie de neurosis o comportamiento disfuncional que ilustramos siguiendo a Perls (1974, 2013), Vásquez Bandín (2005) y Naranjo (1990, 1994) respectivamente:

Cuando los límites entre nosotros y el resto del mundo desaparecen, empieza a corromperse la personalidad. Lo que en un primer momento fue socializante, llegado este punto se convierte en nocivo para el individuo. La razón es simple. Al haber incapacidad de 
diferenciar los esquemas cognitivos y las pautas emocionales que le son propias de aquellas aprehendidas, de manera inconsciente se empiezan a borrar los límites entre nosotros y el medio a un punto tal que se borran los límites que testimonian nuestra esencia y existencia.

El desajuste psicosocial es inminente, y de manera análoga a lo que ocurre a nivel fisiológico con la interrupción o entorpecimiento digestivo, a nivel psicológico no tardan en aparecer signos de desmejora, los cuales son percibidos como obstrucción del proceso de satisfacción de las necesidades con recurrencia en todas las áreas de la vida, obstáculos para lograr el contacto humano, perturbaciones en la caracterización de la personalidad, tendencia a la incoherencia por haber aprendido a no resolver situaciones de satisfacción de necesidades, falta de espontaneidad en la interacción privada y social.

Estamos frente a lo que es llamado en terapia Gestalt perturbaciones neuróticas, cuya aparición busca lograr el balance entre el sujeto y el mundo, a propósito de la demanda social y la incapacidad humana por afrontarla. La neurosis es la respuesta ante la acción social voraz. En consecuencia, el individuo se apropia de mecanismos neuróticos de defensa, siendo la introyección uno de los más destacados. La síntesis que al respecto podemos construir nos indica que el sujeto ante la presión introyecta y se solidariza con las ideas del entorno para minimizar el conflicto aunque en ello pierda su sentido. El proceso, al ser recurrente, desvirtúa la personalidad y lo conduce a un estado patológico de modo permanente.

Con base en lo descrito, consideramos oportuno trasladar la introyección, como categoría analítica para explicar la trama oculta en la formación de competencias metodológicas, donde a nuestro juicio se crea una dinámica análoga a la anteriormente argumentada.

Durante el acto didáctico el novel investigador se ve sometido a tensiones del contexto en atención a los modos de percepción y adopción del método, las cuales clasificamos en dos categorías:

Tensión eugénica frente al conocimiento metodológico: bajo esta denominación encuadramos la interacción crítica entre el facilitador, aprendiz y el conocimiento metodológico. Se reconoce la tensión ante la llegada de un nuevo saber que busca ser organizado en la estructura cognitiva y, con base en ello, se desarrollan los argumentos y contraargumentos que permiten una adecuación diferenciada del conocimiento, atendiendo a la naturaleza y especificidad del objeto en estudio. Visto de esta manera, el saber introyectado es diseccionado en aras de tomar la conveniencia y adaptabilidad del mismo. El investigador se da el permiso de fluir en el método, discriminando los modos operativos más convenientes para la investigación, generando las adecuaciones a las que hubiera lugar, en armonía con su intencionalidad epistémica e identificación metodológica consciente.

Tensión patogénica frente al conocimiento metodológico: en esta categoría se encuentran las respuestas emocionales de tensión y ansiedad del novel investigador ante la imposición del saber metodológico cognitivo y procedimental. Se produce la negación de su criticidad, llegando a convertirse en un migrante cognitivo de su propia creación intelectual. Asimismo, se detecta una respuesta mimética del investigador en formación ante la imposición del facilitador o la institución de adscripción, quienes demarcan la concepción de las acciones metodológicas más convenientes para el desarrollo de las prácticas de investigación que se hacen bajo su radio de influencia.

Esta tensión provoca una especie de abstención epistemológica o invisibilidad del pensamiento crítico del novel en aras de mitigar los conflictos con las figuras representativas o 
institucionales, por ello existe una negación traslapada de su campo fenoménico y epistémico en la aceptación que hace del introyecto. Entre las consecuencias más resaltantes destacan la univocidad operativa al momento de pensar los modos de desarrollar la investigación (un método para todo), el apego irreflexivo a la metódica por encima de la complexión del objeto, la generalización de la investigación como calco y no como un acto de reflexión humana en pro de la inteligibilidad del mundo y, por supuesto, en el peor de los casos, un sentimiento de desconcierto coherente con lo difundido como "todo menos tesis".

De acuerdo con lo manifestado, el manejo del saber metodológico se hace ambivalente en su aprehensión. El novel investigador en intención de aprendizaje experimenta, mediante la interacción discursiva, estados de ansiedad que le invitan a afrontar críticamente el acto investigador, produciendo adecuaciones coherentes con el objeto de estudio. Pero también existe la posibilidad de una respuesta menos ajustada; la de invisibilizarse en su postura epistémica haciendo de los saberes metodológicos introyectados los marcos de la representación de la investigación, obviando el carácter transpuesto de los mismos.

\section{LA TRASPOSICIÓN DIDÁCTICA COMO MARCO EXPLICATIVO DE LA SELECCIÓN INTENCIONAL DE LOS SABERES METODOLÓGICOS}

El concepto de transposición llega al terreno académico por Verret en 1975 (Gómez, 2005) y después, a pesar de la crítica, Chevallard (1998) lo introduce en el contexto de la didáctica de la matemática como teoría de soporte en la construcción del objeto de la enseñanza. Esta perspectiva es la que consideraremos para el establecimiento de nuestra argumentación.

En esta investigación entenderemos la transposición como el proceso de transformación del saber sabio al saber enseñado, caracterizado por la presencia de los siguientes aspectos (Chevallard, 1998):

a. Desincretización del saber: identificación de los elementos parciales que constituyen el objeto de enseñanza, cuya consecuencia más palpable es la descontextualización de los saberes como resultado de hacer una percepción selectiva de los mismos al construir el objeto de la enseñanza.

b. Despersonalización del saber: representa el desapego del saber sabio de su autor. El objeto de la enseñanza se pone al servicio de la comunidad que lo desee. El saber se objetiviza y sale de su creador para entrar a la disciplina.

c. Programabilidad en la adquisición del saber: todo saber enseñado tiene programado su desarrollo. Tiene un carácter evolutivo en cuanto a su nivel de complejidad.

d. Publicidad y control social de la disciplina: sugiere el debate activo del objeto de enseñanza, toda vez que se da a conocer mediante la textualización.

Si bien la teoría de la transposición didáctica tiene un mayor desarrollo y debate, en esta oportunidad solo tomaremos como referente explicativo los elementos que intervienen en la creación del objeto de enseñanza y que ilustramos en las características recién descritas.

Reconsideremos las ideas de Chevallard (1998) en torno a la visión de los saberes sabios como preexistentes, respecto al objeto de la enseñanza y las fortalecemos con lo manifestado por Kant (2007) en cuanto a la existencia del objeto con antelación al sujeto 
y a la capacidad de este de estamparle atributos y propiedades como resultado de la observación. Esto significa que el saber sabio existe antes de que se construya el objeto de la enseñanza, pero también indica que el objeto es vulnerable al observador, quien finalmente lo disecciona en función de sus preconceptos.

Si llevamos esta lógica constructiva del saber didáctico al terreno de la formación metodológica, habrá coincidencia al reconocer, que en el proceso de transformación del saber sabio en objeto de la enseñanza se visualiza un sesgo epistemológico al definir el criterio de demarcación entre lo que es y no ciencia e investigación. Este sesgo también está presente al momento de definir las secuencias operativas de investigación más congruentes con la naturaleza de la cosa en estudio y sus propiedades; un ejemplo de ello se identifica en la transposición de la investigación realizada desde el Círculo de Viena y la Escuela de Frankfurt.

En ambos casos, existen referencia a los procesos de investigación y a su rol en la sociedad y crecimiento de la ciencia. Los vieneses, bajo la dirección de Carnap y Reichenbach construyen la investigación como objeto a enseñar a partir del lema "unificación de la ciencia", teniendo como referentes a la filosofía empírica y una actitud de negación ante la existencia de la metafísica. El objeto a enseñar es coherente con las características siguientes (Alvarez, 1999; Quezada, 2004): a) uso de la lógica y matemática como vía para comunicar y proceder en el marco de la ciencia, b) la investigación debe estar apoyada con la evidencia empírica, c) el contenido de verdad se cuantifica a fin de poder someter a confirmación las hipótesis generadas, d) presencia de criterios justificacionistas del problema construido a partir de la percepción interaccional de las propiedades del objeto que se estudia. En síntesis: el conocimiento pasa por un proceso de rigurosidad y control absoluto, donde cada una de sus fases deben ser operacionalizadas y luego articuladas con el máximo control posible para garantizar su verificabilidad.

Para el caso de los frankfurtianos, el conocimiento es el resultado de la interacción de condiciones históricas, dialécticas, ideológicas. La ciencia y la investigación son medios para la emancipación humana y transformación social, por tanto, se distancia de los valores de asepsia que le imprimen los seguidores del Círculo de Viena. La intencionalidad de este grupo académico es comprender y cuestionar las ideologías y representaciones que oprimen a los actores sociales y condicionan sus procesos de significación y cambio a nivel personal y colectivo (Healy, 2001; Muñoz, 2005).

En ambos casos el saber sabio es el mismo: la ciencia y la investigación, pero su transformación en saber para ser enseñado las muestran como dos elementos distintos. En este punto se suma una evidencia adicional para la comprensión del rol de la transposición en la configuración de los introyectos.

Al momento de realizar la abstracción del saber sabio y activar su metamorfosis, se fijan las perspectivas epistemológicas regentes de la comunidad científica involucrada en la selección y caracterización de ese objeto a enseñar, concretado en la transposición externa, que más tarde se hace interna cuando el formador desmiembra el objeto construido y termina enseñándolo en el aula o durante la tutoría, poniéndole un poco de su epistemología personal.

Se tiende una brecha entre el saber sabio, en este caso, el conocimiento de la práctica investigadora y lo que termina llegando al aula y al novel investigador. Esta brecha existe, porque la perspectiva del que transpone tiene una motivación y un requerimiento a satisfacer (De Faría, 2006). En la selección del contenido se hace presente la voluntad de poder del que transpone y, con ello, la ideología que se aspira instalar. 
Como puede percibirse, la naturaleza de la transposición gesta una brecha entre el saber sabio y el objeto de la enseñanza, situación que activa reclamos sobre la autenticidad de lo que se plasma en el currículo y la intencionalidad de su inserción. Lo que llega al aula suele estar tan filtrado y descontextualizado, que el proceso de aprenderlo reclama la exportación de un contexto para comprender la interacción de las lógicas transpuestas. Pareciera paradójico, pero los actos humanos al hacerlos objetos de enseñanza se cosifican, y luego para enseñarlos se reclama la vuelta a su esencia para que el humano aprenda lo que un día salió de él.

Justamente este efecto de la transposición genera a nuestro parecer tres comportamientos didácticos en los formadores de investigadores nóveles, los cuales describimos a continuación:

a. Identificación con la pauta metódica curricular: el formador se adhiere a las prescripciones curriculares. Se dedica a enseñar metodología desde la perspectiva enunciada en el currículo. La adhesión se fundamenta en la creencia de que el currículo prescribe los saberes requeridos por el formando para llegar a ser investigador y producir conocimiento válido y confiable. Por tanto, solo es lícito lo que se ajuste al esquema referido en la estructura programática del proceso formativo. Dentro del currículo, todo; fuera de él, no es posible conocer la investigación ni aprenderla.

b. Modificación de la pauta curricular con base en su voluntad de poder: en esta perspectiva se ilustra el comportamiento docente dirigido a transformar a voluntad y con base en la experiencia personal los modos de concebir y hacer investigación. Si bien se aceptan prescripciones curriculares, las creencias del formador y sus epistemologías personales terminan imponiéndose en la concepción, diseño e instrumentalización de los procesos de investigación científica.

c. Contextualización del saber enseñado: En esta postura el formador muestra opciones estructurantes y dinamizadoras del hacer investigador. Existe el reconocimiento de la diversidad al momento de estructurar y operativizar los procesos de investigación; sin embargo, la formación pasa por hacer usos diferenciados de los saberes sabios y convertirlos en objetos de enseñanza en virtud de los requerimientos del grupo que demanda de esas competencias. Esta perspectiva ofrece la posibilidad de poner el conocimiento metodológico al servicio del investigador y sus inquietudes.

Se nota, al igual que en la introyección, que la transposición es un tema irrenunciable cuando de formar investigadores se trata. No obstante, la adherencia fiel a lo transpuesto se constituye en un riesgo. Recordemos que el contexto por su carácter dinámico y caótico hace que el objeto didáctico caduque de manera constante, de allí que adherirse a lo transpuesto de una manera acrítica genera la instalación de competencias metodológicas en los formandos que, más allá de ser aliados en el desarrollo del estudio, se constituyen en potenciales obstáculos para su concreción.

\section{ENCUADRE METODOLÓGICO}

El estudio es de tipo descriptivo en la modalidad documental (Fontaines, 2012) debido a que se establece una caracterización del comportamiento diferencial de las 
secuencias operativas de investigación observadas en un total de 220 trabajos de titulación presentados por los estudiantes de pregrado en la Unidad Académica de Ciencias Sociales de la Universidad Técnica de Machala, durante el año 2014. La recolección de los datos se realizó mendiante el desmontaje de la estructura operativa del trabajo de titulación. De manera puntual, los trabajos se interpelaron en torno a los siguientes elementos: formas de concepción operacional del objeto de estudio, modos de representación de las variables, naturaleza de los diseños de investigación, concepción y abordaje de la recolección y análisis de los datos. La obtención de los resultados se llevó a cabo mediante el análisis inductivo del contenido (Krippendorff, 1990; Reguera, 2008) a fin de poder estimar las macro categorías que testimonien la naturaleza semántica de los introyectos y su representación en los trabajos de titulación.

\section{HALLAZGOS DEL ESTUDIO: EL CONTENIDO DE LOS INTROYECTOS EN LOS TRABAJOS DE TITULACIÓN}

Los resultados del análisis de contenido hecho sobre la significación que ostentaron las categorías en estudio permitieron la identificación de los siguientes introyectos metodológicos:

\subsection{INTROYECTO: EL MÉTODO ESTÁ POR ENCIMA DE LAS DIFERENCIAS ESTRUCTURALES Y FUNCIONALES DEL OBJETO}

La idea identificada sugiere que toda investigación debe ser abordada desde una única perspectiva metodológica. En el caso estudiado, el 100\% de los trabajos de titulación, sin distingo de la carrera de adscripción, siguieron secuencias operativas identificadas con el enfoque epistemológico empírico inductivo también conocido como positivista-cuantitativo (Fontaines, 2010, 2012; Padrón, 1998). Para que el trabajo sea objeto de valoración debe ser procesado según el guion establecido, el cual valora la inducción como vía de acceso al conocimiento, el uso del lenguaje fisicalista-matemático, la operacionalización de variables como signo del atomismo lógico, el empleo de la verificación como criterio de demarcación.

A partir de este introyecto, se produce la emisión de juicios respecto a la cientificidad y valor de unos estudios sobre otros. En tal sentido, solo es científico aquello que sea congruente con el saber transpuesto. Justamente, el carácter excluyente de esta idea, violenta la naturaleza plural de la práctica de la investigación al tener que tallar los objetos a la medida del método.

Para ilustrar esta idea le invitamos a pensar una investigación que busque determinar la representación e intensidad del dolor de padres que han perdido a sus hijos por acción de la inseguridad urbana. Estando bajo los efectos del introyecto, tanto los investigadores como sus evaluadores generarán alternativas para poder conceptualizar operacionalmente el objeto de estudio, aunque esto signifique su descontextualización por no poder aprehender desde lo cuantitativo la energía emocional que se solapa en los diversos recursos discursivos de los que el ser humano hace uso para representar su situación emotiva-afectiva. Esta ilustración busca reflejar cómo el objeto es vulnerado por el método mientras este último, que inicialmente es concebido como un medio, se convierte en un fin en sí mismo. 


\subsection{INTROYECTO: IMPOSICIÓN ARQUETÍPICA DE LA DIRECCIÓN DE LAS VARIABLES}

Este introyecto justifica la presencia de una dirección previa en las variables que se estudian. En este caso particular se ilustra una permanente relación de dependencia, obviando los vínculos de asociación o de estudio que se dan en las variables. De manera puntual, se resalta el esfuerzo del investigador por encontrar variables independientes y dependientes en estudios donde no hay modo de visualizar tal esquema lógico. Por ejemplo, si estudiamos la relación existente entre la autoeficacia y resiliencia de estudiantes universitarios, no podríamos establecer que la autoeficacia pueda ser una variable dependiente y que la resiliencia fuese independiente o lo contrario. En todo caso las variables tienen implicadas una relación de asociación que se obvia por la necesidad de dar respuesta a la impostura del introyecto.

En tal sentido consideramos importante establecer que la percepción de los atributos o variables en el objeto de estudio son cambiantes, en atención al ángulo de análisis tal como se explica desde el concepto de paralaje (Žižek, 2006) y, por tanto, resulta inviable pretender una permanente dependencia, donde puede fluir otro tipo de vínculos que garantizan una mayor estabilidad y consistencia interna de los estudios que se emprenden.

\subsection{INTROYECTO: LOS DATOS SE DEBEN EVALUAR ESTADÍSTICAMENTE PARA GARANTIZAR CIENTIFICIDAD EN EL ESTUDIO}

Este introyecto es signo de vigencia del saber transpuesto por el Círculo de Viena, a pesar de tener más de cien años. En el total de los trabajos de titulación observados, los datos solo hablan mediante la traducción estadística, como criterio de nulidad de subjetividad en la emisión de los resultados. Este concepto deja de lado los valores que desde el contexto de descubrimiento incrementan la dinámica de contextualización de los datos y, al mismo tiempo, mecaniza la relación de interacción con el objeto mediante técnicas e instrumentos que, de manera prescriptiva, promuevan la emisión de respuestas susceptibles de ser convertidas en valores numéricos. Ejemplo de ello es el uso recurrente de encuestas con formato Likert como el instrumento clave para la recolección de los datos y su posterior emisión en lenguaje numérico.

Esta acción en el terreno particular de las ciencias sociales, devela una visión reduccionista de la realidad en estudio, ya que los procesos humanos son tan variables como caóticos. Construir una escala supone transponer conductas esperadas y movilizar el acuerdo del respondiente en virtud del marco de referencia del autor del instrumento. Sin embargo, el sujeto de evaluación puede estar en posesión del atributo, pero el mismo se camufla desde otras representaciones discursivas no representadas en la semántica de los reactivos que componen el instrumento de medición. Por tanto, es imperativo que existan cambios a favor de la percepción multinivel del dato para de esta manera, llegar a comprender la integralidad de lo estudiado.

\subsection{INTROYECTO: VALORACIÓN SESGADA DEL PARADIGMA}

El contenido de este introyecto sugiere que la visión epistemológica empírica, por ser el soporte de la secuencia metodológica identificada en el total de los trabajos de titulación, 
presenta condiciones estructurales y funcionales que le imprimen un carácter mesiánico si de lograr productos científicos se trata. Si bien se reconoce la presencia de otros enfoques y posiciones teóricas, los mismos no son considerados como ejes estructurantes de las prácticas metodológicas, generando argumentos que enaltecen, en grado superlativo, las bondades de este paradigma sobre los otros.

Este introyecto devela un tipo de anclaje epistémico que inhibe el acercamiento a la complementariedad metodológica para generar respuestas más ajustadas a las demandas del objeto y de la investigación como un todo (Burke y Onwuegbuzie, 2004; Creswell y Piano, 2007). Consideramos que las consecuencias más tremendas de esta situación serían el desconocimiento de la pluralidad del objeto y la legitimación de las constricciones en su abordaje.

\subsection{INTROYECTO: TODA INVESTIGACIÓN DEBE LLEVAR APAREJADA UNA PROPUESTA}

En este introyecto se reconoce que la completitud de la investigación se designa por la construcción de una propuesta, así lo develan el total de los trabajos de titulación observados. Sin propuesta la investigación está inconclusa y, por lo tanto, no se puede valorar. Enmarcados en este introyecto, los objetivos de la investigación pasan a tener un rol ornamental en el trabajo. Solo marcan dirección y no límites. En todo caso, es bueno recordar que la idea del objetivo es llegar a delinear el alcance el estudio, de allí que si en la investigación se pretende establecer la relación entre "a" y "b", no tendría sentido llegar a realizar propuestas, porque no es la intencionalidad del mismo.

La información manejada testimonia cómo la construcción de ideas o representaciones que circulan en el ambiente de la formación investigadora, inhiben el contacto claro y direccionado entre el novel investigador y el conocimiento del que se empodera. Investigar es un proceso de aperturas no de constricciones, es una dinámica que permite develar estructuras y relaciones entre aspectos del mundo que de momento han pasado desapercibidos para el hombre, de allí que constreñir las vías de acceso solo aleja a potenciales investigadores del camino de comprender las tramas que subyacen a su naturaleza.

\section{REFLEXIONES FINALES}

El problema del conocimiento y sus formas de aprehensión fueron impulsados a partir del discurso del método (Descartes, 2000). En esta obra se declara un saber transpuesto que tendrá resonancia a lo largo de muchos siglos. No obstante, su presencia abrió debates que llevaron al reconocimiento de la posición diferenciada del sujeto frente al objeto de estudio (Cornford, 2011). La idea de que el objeto influye en el hombre para transmitirle sus cualidades y la búsqueda de un principio —ả $\chi \chi \eta ́$ - que explicara el origen de la realidad existente (Kirk, Raven y Schofield, 2011), cambió radicalmente con el surgimiento del giro kantiano que termina reconociendo al sujeto como el que determina las condiciones del objeto en atención a lo observado (Kant, 2007).

El hombre adoptó la posición de dominador del conocimiento y la ciencia para dar sentido a la realidad circundante — dejó de sentirse e interpretarse como parte del entorno natural y se situó como amo de todo_- pero en ese "progresar" en sentido lineal, "hacia el frente", se olvidó de la existencia de otras posibilidades, es decir, se cerró a la diversidad 
de formas o modos de proceder para obtener los mismos resultados, debido a que parecían "escandalosas" las afirmaciones diferentes a las establecidas como científicas (Kuhn, 2004) o porque la corroboración de dicho conocimiento no podía ser medible o cuantificable.

Progresivamente y con el avance de las ciencias exactas y naturales con Newton, Einstein, Darwin, entre otros, el método dejó de ser un medio para convertirse en un fin en sí; dejó de ser la herramienta que servía para la consecución de resultados en investigación y pasó a ser la razón de la investigación más allá de lo que quisiese lograrse. Esta transformación, de medio en fin, ha dado como resultado serias desavenencias en el plano de la investigación: todo aquello que no siga un procedimiento "consagrado" será considerado anárquico y sin valor científico, rasgo que aún se mantiene presente en los trabajos de titulación observados.

En la misma línea y con el afán de conseguir "categoría científica" las ciencias humanas o sociales han robustecido este introyecto de medir todo para que se convierta en una certeza y, con esto, se han alejado de la pluralidad que trasciende lo medible o cuantificable. $\mathrm{Al}$ parecer se ha heredado de la transposición didáctica que la única forma de hacer investigación es la que se puede ver reflejada en números, gráficos o cuadros: el método se ha convertido en un dogma apodíctico que no acepta argumentos en contra a lo que disponga y esta dogmatización está presente en todos los planos académicos (Ricoeur, 2006).

Finalmente, la dogmatización de un método en detrimento de las partes previa y posterior del proceso de investigación —idea y praxis o resultados- distorsionará profundamente la búsqueda de certezas cuantitativas o cualitativas. El adherirse ritualmente a un método imposibilitará el descubrimiento del movimiento existente en toda la realidad circundante,

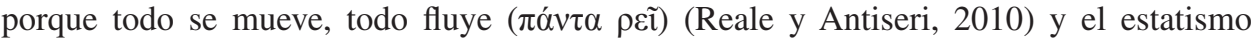
monolítico en un método dará como resultado pobres y limitadas investigaciones, de allí la necesidad de empoderar criterios de libertad y adecuación metodológica en los formandos de la UTMach a fin de que puedan abordar integralmente el objeto de estudio sin distingo de su tipo, pero atendiendo a su singularidad.

\section{REFERENCIAS BIBLIOGRÁFICAS}

Alvarez, F. (1999). El problema de la verdad: una aproximación analítica. México: Universidad Iberoamericana.

Arros, M., \& Valenzu, F. (2006). Teoría psicoanalítica de la depresión: Una revisión de distintas propuestas para su comprensión y clasificación. Revista Gaceta universitaria, 2(4), 473-481.

Bornmann, L. (2011). Mimicry in science? Scientometrics, 86, 173-177.

Bunge, M. (2004). Epistemología. Barcelona: Siglo XXI editores.

Burke, R., \& Onwuegbuzie, A. J. (2004). Mixed methods research: A research paradigm whose time has come. Educational Researcher, 33(14), 13-26.

Camacho, H. (2001). Enfoques epistemológicos y secuencias operativas de investigación. Maracaibo: Universidad Rafael Belloso Chacín.

Caparrós, E. (2012). Competencia comunicativa para la acción didáctica. In J. Roser y R. Rickenman, Diálogos sobre investigación de la acción didáctica conjunta. Retos y perspectivas (pp.77-110). España: Universidad de Girona.

Chevallard, Y. (1998). La transposición didáctica. Del saber sabio al saber enseñado (3era ed.). Argentina: Aique.

Cornford, F. (2011). Antes y después de Sócrates. Barcelona: Ariel. 
Creswell, J., \& Piano, V. (2007). Designing and Conducting Mixed Methods Research. California, EE.UU.: Sage Publications.

De Faría, E. (2006). Transposición didáctica: definición, epistemología, objeto de estudio. Cuadernos de investigación y formación en educación matemática, 1(2), 1-12.

Descartes, R. (2000). Discurso del Método. Madrid: Alianza Editorial.

Echeverría, J. (1995). Filosofía de la ciencia. España: AKAL.

Feyerabend, P. (1986). Tratado contra el método: esquema de una teoría anarquista del conocimiento. España: Tecnos.

Feyerabend, P. (2008). Adiós a la razón. España: Tecnos.

Fontaines, T. (2010). Integración metodológica en el proceso de investigación en ciencias sociales, una aproximación teórica. Revista Estudios Digital, Número Especial. Recuperado desde http:// www.revistaestudios.unc.edu.ar/articulos03/dossier/4-fontaines.php

Fontaines, T. (2012). Metodología de la investigación. Caracas: Júpiter editores.

Fontaines, T., \& Rodríguez, Y. (2008). Estructuras e interacciones en la construcción del conocimiento científico. Una aproximación a partir de los planteamientos teóricos de Piaget y Vigotsky. Laurus, revista de educación, 14(28), 97-121.

Gadamer, H. G. (2007). Arte y verdad de la palabra. España: Paidos.

Giroux, H. (1990). Los profesores como intelectuales. España: Paidos.

Gómez, M. A. (2005). La transposición didáctica: historia de un concepto. Revista latinoamericana de estudios educativos, 1, 83-115.

González, J. J., Simo, J., Gamietea, C., Caudillo, C., \& Zarco, S. (2005). Concepto de padres combinados. Revista latinoamericana de psicopatología, 8(1), 64-72.

Gutiérrez, M. (2013). Sándor Ferenczi y la intelectualidad húngara del siglo XX. Affectio Societatis, $10(18), 1-12$.

Habermas, J. (2008). El discurso filosófico de la modernidad. Argentina: Katz editores.

Healy, K. (2001). Trabajo social: perspectivas contemporáneas. España: Morata.

Henwood, K. (2005). Reinventing validity: Reflections on principles and practices from beyond the quality-quantity divide. In Z. Todd, B. Nerlich, S. McKeown, \& D. Clarke, Mixing Methods in Psychology. The integration of qualitative and quantitative methods in theory and practice (pp. 37-57). New York, EE.UU.: Psychology Press is a part of the Taylor \& Francis Group.

Hesse, S. N. (2010). Mixed Methods Research. New York, EE.UU.: The Guilford Press.

Hough, M. (1999). Técnicas de orientación psicológica. España: Narcea editorial.

Husserl, E. (1998). Invitación a la fenomenología. España: Paidos.

Johsua, S., \& Dupin, J. (2005). Introducción a la didáctica de las ciencias y la matemática. Buenos Aires: Ediciones Colihue.

Kant, I. (2007). Crítica de la razón pura. Buenos Aires: Colihue.

Kirk, G. S., Raven, J. E., \& Schofield, M. (2011). Los filósofos presocráticos (3era ed.). Madrid: Gredos.

Krippendorff, K. (1990). Metodología de análisis de contenido. España: Paidos.

Kuhn, T. (2004). La estructura de las revoluciones científicas (8ava ed.). México: Fondo de Cultura Económica.

Lakatos, I. (2007). La metodología de los programas de investigación cientíica. España: Alianza editorial.

Lakatos, I. (2011). Historia de la ciencia y sus reconstrucciones racionales. Madrid: Tecnos.

León, O. (2008). La ciencia y la tecnología en la sociedad del conocimiento. Ética, política y epistemología. México: Fondo de Cultura Económica.

Merton, R. (1996). On social structure and science. EE.UU.: The University of Chicago Press.

Moynihan, M. (1968). Social Mimicry. Character Convergence Versus Character Displacement. Society for the Study of Evolution, 22(2), 315-331.

Muñoz, B. (2005). Modelos culturales. Teoría sociopolítica de la cultura. México: Anthropos. 
Naranjo, C. (1990). La vieja y novísima Gestalt. Chile: Cuatro Vientos.

Naranjo, C. (1994). Carácter y neurosis. Una visión integradora. Chile: Comunicaciones Norestw.

Nietzche, F. (2000). La voluntad de poder. Madrid: Edaf.

Outhwaite, O. (1994). Habermas. A critical introduction. California: Stanford University Press.

Pacheco, F., \& Pons, S. (2003). Transferência como experiência do vivido e transmissão psíquica: a herança de Sándor Ferenczi. Pulsional Revista de Psicanálise, 164, 17-26.

Padrón, J. (1998). Modelo de variabilidad de la Investigación educativa. Caracas, Venezuela: Universidad Nacional Experimental Simón Rodríguez.

Pasteur, G. (1982). A Classification Review of Mimicry Systems. Annual Review of Ecology and Systematics, 13, 169-199.

Perls, F. (1974). Sueños y existencia. Chile: Editorial Cuatro Vientos.

Perls, F. (1997). El enfoque gestáltico. Testimonio de la terapia. Santiago de Chile, Chile: Cuatro Vientos Editorial.

Perls, F. (2013). El enfoque de gestalt y testigos de la terapia (20ma ed.). Santiago de Chile: Editorial Cuatro Vientos.

Popper, K. (1972). Conjeturas y refutaciones. El desarrollo del conocimiento científico. España: Paidos.

Popper, K. (1982). La lógica de la investigación científica. Madrid: Tecnos.

Prigoggine, Y. (1997). El fin de las certidumbres. Chile: Editorial Andrés Bello.

Prigoggine, Y. (2009). Las leyes del caos. España: Editorial Crítica.

Quezada, F. (2004). Aproximación a la metodología de la ciencia. Las ciencias sociales y la contabilidad. España: Ediciones de la Universidad Castilla de la Mancha.

Reale, G., \& Antiseri, D. (2010). Historia del Pensamiento Filosófico y Científico (Vol. I). Barcelona: Herder.

Reguera, A. (2008). Metodología de la investigación lingüística. Prácticas de escritura. Argentina: Brujas.

Ricoeur, P. (2006). A hermenêutica bíblica. São Paulo: Loyola.

Rycroft, C. (2010). ¿Por qué los analistas necesitan la transferencia de sus pacientes? Clínica e investigación relacional, 4(1), 46-52.

Sainsbury, M. J. (1978). Introducción a la psiquiatría. Madrid: Morata.

Sanchez, H. I. (2010). Proyección, identificación, identificación proyectiva. Joseph Sandler, 1989. Apertura psicoanalítica. Revista internacional de psicoanálisis, 36. Recuperado desde: http:// www.aperturas.org/articulos.php?id=0000662\&a=Proyeccion-Identificacion-IdentificacionProyectiva-Joseph-Sandler-1989

Santos, E. (2014). El mundo natural según la teoría de la relatividad de Einstein. España: Ediciones de la Universidad de Cantabria.

Vasquez Bandín, C. (2005). Terapia Gestalt. In J. L. Romero Cuadra, \& R. Alvarado Rafael, Psicópolis (pp. 166-182). España: Kairos.

Vigotsky, L. (2004). Teoría de las emociones. Estudio histórico psicológico. Madrid: Akal.

Villacañas, L. S. (2013). Giro copernicano y ciencias sociales. España: Plaza y Valdés.

Žižek, S. (2006). Visión de paralaje. Buenos Aires: Fondo de Cultura Económica. 
